\title{
Improvement in Optical Properties of Nuclear Track Detector
}

\author{
Doaa Hassan Shabaan ${ }^{1,2, ~ *, ~ T a y s e e r ~ I b r a h i m ~ A L-N a g g a r ~}{ }^{1,3}$ \\ ${ }^{1}$ Department of Physics, Faculty of Women for Art, Science and Education, Ain Shams University, Cairo, Egypt \\ ${ }^{2}$ Department of Physics, Faculty of Science and Arts, Jazan University, Jazan, Saudi Arabia \\ ${ }^{3}$ Department of Physics, Faculty of Science and Arts, Najran University, Najran, Saudi Arabia
}

Email address:

do_tahaa@yahoo.com (D. H. Shabaan)

${ }^{*}$ Corresponding author

\section{To cite this article:}

Doaa Hassan Shabaan, Tayseer Ibrahim AL-Naggar. Improvement in Optical Properties of Nuclear Track Detector. Nuclear Science. Special Issue: Radiation Dosimetr. Vol. 4, No. 2, 2019, pp. 23-26. doi: 10.11648/j.ns.20190402.12

Received: July 3, 2019; Accepted: July 31, 2019; Published: September 2, 2019

\begin{abstract}
The purpose of this work was obtaining information about the interaction of $\gamma$ - ray with CR-39 track detector by using the UV-Vis spectrometry and FTIR which can be used in concerning sensor for gamma irradiation. CR-39 samples irradiated by radioactive source Co-60 at different doses are $(0,100,200,300,400$, and $500 \mathrm{kGy})$. The UV-Vis spectroscopy show transitions electronic in the visible region from ground state to excited state, by increasing gamma doses, and the absorbance spectrum for all samples take the same behavior with slightly shift. This shift due to decrease in the optical band gap energy $E_{g}$. The FTIR spectra show for all samples there are increases in the intensity of the characteristic peaks with increasing gamma ray, at 3234,2367 and $1817 \mathrm{~cm}^{-1}$, this increase may be related to more oxidation process that, in turn was produced on the polymer chains by increase gamma doses, and the number of peaks at $1817,2367,2645$ and $3234 \mathrm{~cm}^{-1}$ is belong to carbonate group $\mathrm{C}=\mathrm{O}$ stretching vibration, $\mathrm{O}=\mathrm{C}=\mathrm{O}$ asymmetric by stretching, $\mathrm{C}-\mathrm{H}$ Stretching, $\mathrm{H}_{2} \mathrm{O}$ free stretching vibration, respectively. By increase gamma ray did not observed changes in the CR-39 groups but observed change in the intensities of peaks, then CR-39 detector can be used properly in the field of radiation dosimetery.
\end{abstract}

Keywords: CR-39 Polymer, UV/Vis, FTIR, Radiation Dosimetry, Gamma Ray Irradiation

\section{Introduction}

Solid state nuclear track detector CR-39 has a chemical formula $\left(\mathrm{C}_{12} \mathrm{H}_{18} \mathrm{O}_{7}\right)$ and it advantages by perfect structural stability and it has high resistance to most of the solvent, chemicals and different environmental factors. CR-39 is clear in the visible range and almost entirely no transparent in the electromagnetic range [1]. It has the track registration property because of high detection efficiency, high sensitivity, and short etching time. This polymer is also an excellent material for a number of industrial, medical and optical applications [2]. CR-39 plastic detector is one of the most commonly used for radiation detection, because its high sensitivity to protons and alpha particles. These particles do not caused tracks but can make weighty and sometimes intense effect on the properties of track [3-4]. The primary configuration of solid-state nuclear track detectors (SSNTD) such as CR-39 polymer destroys by the interaction of radiation with it, as well as heavy chemical variations take place in polymers under the effect of ionizing radiations (Xrays, gamma rays, fast and slow neutrons, fast electrons, alpha particles, protons, and other products of nuclear reactions) [5]. Form point of view the process of destruction makes by severance, cross-linking and release of molecules, atoms, and molecular fragments, this due to changes in some properties of SSNTD polymer like conductivity, density, optical absorption, molecular-weight distribution etc [3]. Polymer substances have decay by random fracture of the original chain with the amount of fractures being relative to the radiation dose which is surly related to the effect of $\mathrm{x}$ rays or gamma rays on polymer. The bond fracture may provide rise to free radicals ionic species, water molecules, gaseous products, etc [6]. When CR-39 polymer was irradiated by gamma ray or $\mathrm{x}$-ray, $\mathrm{Co}_{2}$ molecules are formed and the formation of $\mathrm{CO}_{2}$ was attributed to bond breaking in 
CR-39 polymer by the effect of radiation doses. This $\mathrm{Co}_{2}$ is trapped inside, this can result the successive crazing and cracking of the plastic due to collected local stress. The production of $\mathrm{Co}_{2}$ is a function of gamma ray dose [6-7]. The study of the physico-chemical properties like optical and chemical properties of the polymer after the effect of radiation on CR-39 polymer are very important because these parameters are modified after exposed of polymer to radiation dose and also these changes may enhance the polymer applications in many fields [8]. The target of the present work is to produce the response of CR-39 polymer to different gamma doses from $(0-500 \mathrm{kGy})$, and study the optical and chemical properties of CR-39 polymer under these gamma doses.

\section{Experimental Details}

\subsection{Material}

Is this work, the poly ally diglycol carbonate detectors named CR-39, were cut from sheet manufactured by TASRAK Analysis System, Ltd., UK: TASTRACK. The CR39 has a chemical formula of $\mathrm{C}_{12} \mathrm{H}_{18} \mathrm{O}_{7}$. The samples have thickness $1 \mathrm{~mm}$ and dimension $2 \mathrm{~cm} \times 2 \mathrm{~cm}$. The molecular structure of the monomer CR-39 polymer track detector show in in figure 1.<smiles>C=CCOC(=O)OCCOCCOC(=O)OCC=C</smiles>

Figure 1. The structure of CR-39 monomer.

\subsection{Irradiation}

CR-39 samples were irradiated with different doses are $(0$, $100,200,300,400,500 \mathrm{kGy})$ of gamma ray by using radioactive point source of Co-60 has half-life $5.27 \mathrm{yrs}$, and energies 1.174, 1.332 Mev.

\subsection{UV/Vis Absorption}

The change in optical absorption of pristine and irradiated CR-39 detector with gamma ray were obtained by UV/V is Spectra which was carried out by UV/Visible Double Beam spectrometer JASCO V-630 in the range of wavelength 200$1100 \mathrm{~nm}$ at an interval of $1 \mathrm{~nm}$, at ambient condition.

\subsection{Fourier Transforms Infrared Spectroscopy (FTIR) Spectroscopy}

The functional groups in molecules of CR-39 can be identified by FTIR Spectroscopy. The spectra for all CR39 samples were obtained by Nicolet 6700 spectrometer, thermo Scientific, in the wavenumber range from 400$4000 \mathrm{~cm}^{-1}$ and resolution of $4 \mathrm{~cm}^{-1}$. The spectra was recorded to obtain the variation in the functional groups of CR-39 between the pristine sample and the irradiated with gamma rays.

\section{Results and Discussion}

The poly-ally chain connected by di-ethylene glycol dicarbonate link form structure for CR-39 polymer. This structure containing three main functional groups: ally group, carbonyl group and ether group, which are responsible for the improvements in physicochemical properties after radiation exposure, so the presence of allyl group in the CR-39 structure makes it more sensitive for any type of radiation [2-9].

\subsection{UV/Vis Studies}

The transitions electronic in the visible region for the absorption spectra from the ground state to the excited state have been studied by UV-Visible technique. Figure 2. shows the variation in the absorbance of pristine and irradiated CR39 detector samples with the wavelength at different doses: a) 0kGy, b)100 kGy, c)200 kGy, d)300kGy, e)400 kGy, f) $500 \mathrm{kGy}$, and it is shown that the absorbance spectrum for all samples take the same behavior with slightly shift by increasing gamma doses. This shift denoted to the decrease in the optical band gap energy $E_{g}[10]$, this behavior is correlated with the formation of carbon [11]. This result confirms that the influence of gamma ray on CR-39 polymer detectors are affected in the UV-Visible region, which is consistent with the pervious results [12-13].
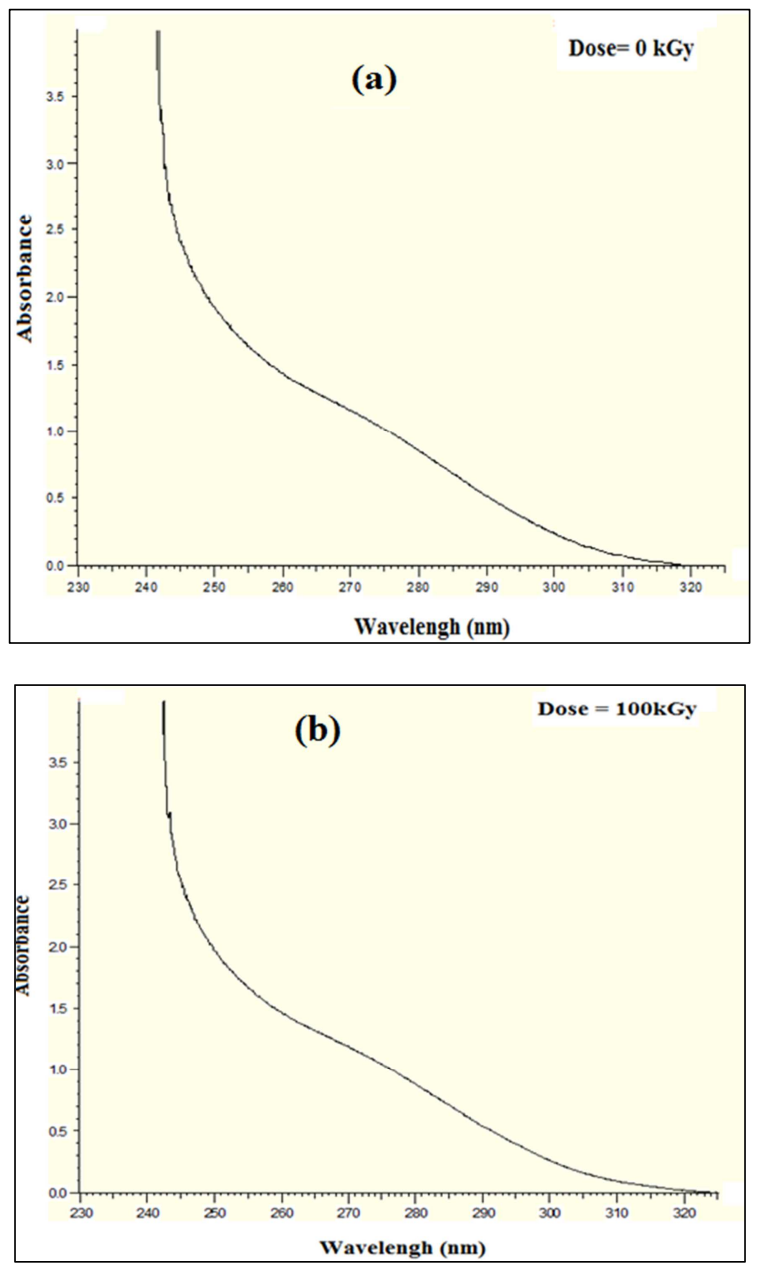

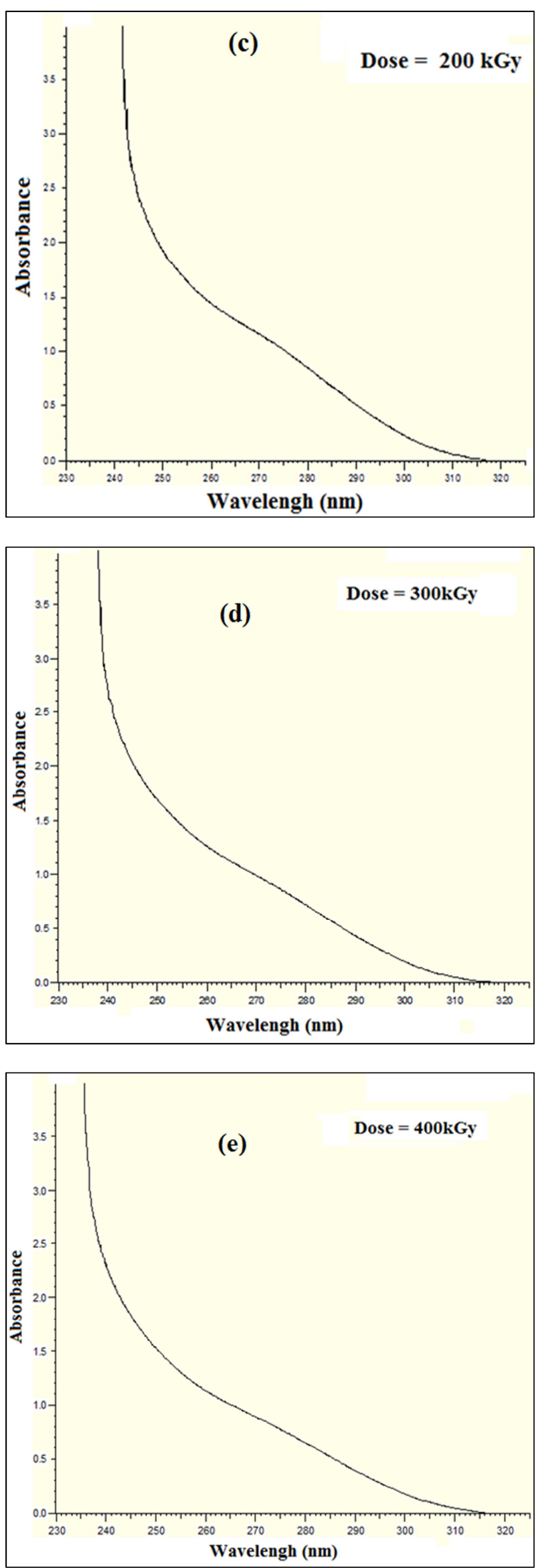

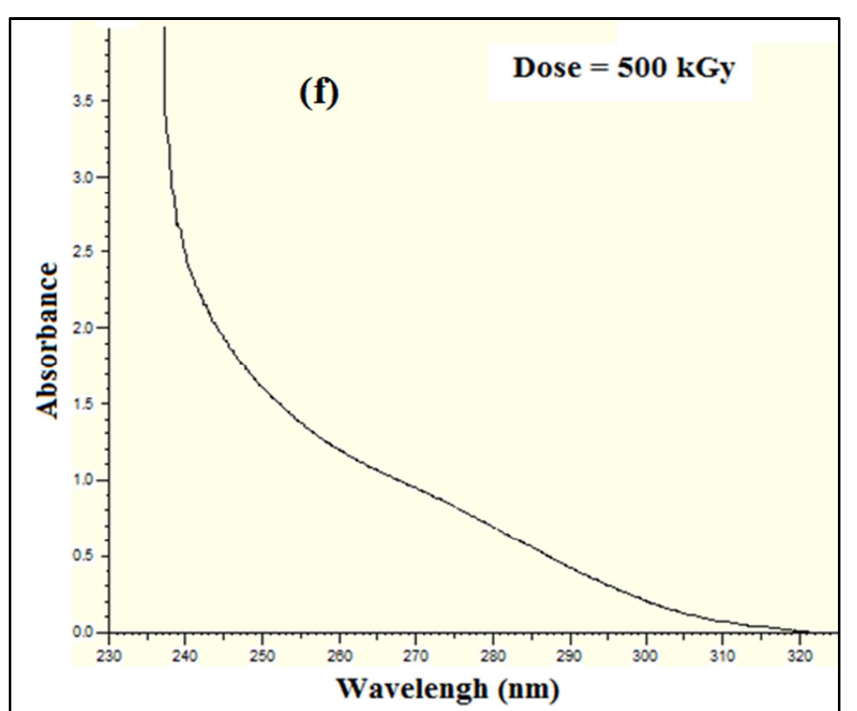

Figure 2. $U V$-visible spectra of the variation in the absorbance at a) $0 \mathrm{kGy}$, b) $100 k G y$, c) $200 k G y$, d) $300 k G y, e) 400 k G y, f) 500 k G y$, with the wavelength.

\subsection{FTIR Spectroscopy}

Figure 3 shows the spectra of FTIR for pristine and irradiated CR-39 sample detector with doses 0,100, 200, $300,400,500 \mathrm{kGy}$. It is clear that the FTIR spectra for all samples are characterized by number of peaks at 1817 , 2367, 2645 and $3234 \mathrm{~cm}^{-1}$ is belong to carbonate group $\mathrm{C}=\mathrm{O}$ stretching vibration, $\mathrm{O}=\mathrm{C}=\mathrm{O}$ asymmetric stretching, C-H Stretching, $\mathrm{H}_{2} \mathrm{O}$ free stretching vibration, respectively [14]. After irradiation, there is no shift in the peak position or formation of new bonds, there are increases in the intensity of the characteristic peaks with increasing gamma ray, at 3234, 2367 and $1817 \mathrm{~cm}^{-1}$. This increase may be related to more oxidation process that, in turn was produced on the polymer chains by increase gamma doses [15]. In general, did not observed change in the CR-39 groups under gamma irradiation, but observed changes in the intensities of peaks.

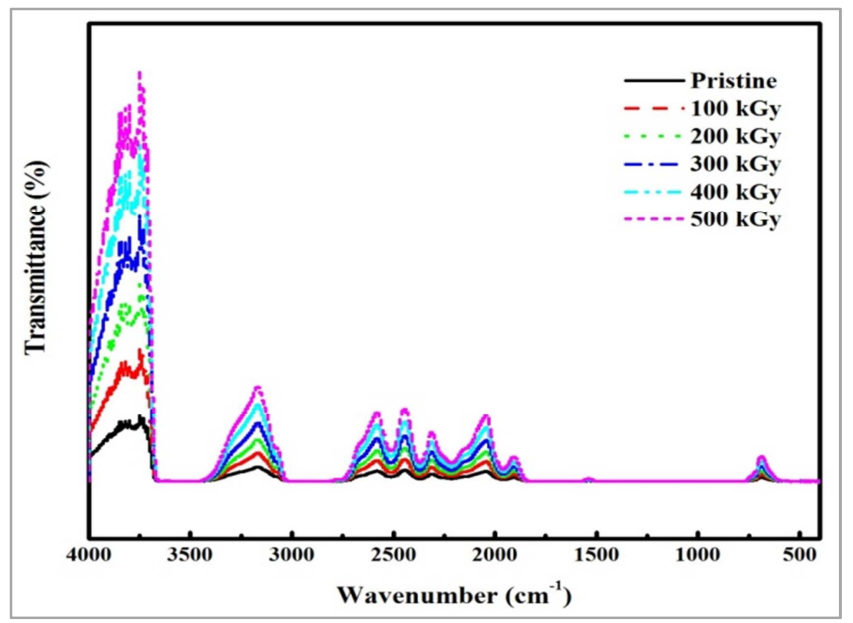

Figure 3. Spectra of FTIR for pristine and irradiated CR-39 polymerwith different doses of gamma rays. 


\section{Conclusion}

The UV-Vis spectroscopy show transitions electronic in the visible region from ground state to excited state, by increasing gamma doses the absorbance spectrum for all samples take the same behavior with slightly shift. This shift due to decrease in the optical band gap energy $E_{\mathrm{g}}$. Thus irradiation makes the polymer more conductive, by increase gamma ray did not observed changes in the CR-39 groups but observed change in the intensities of peaks.

\section{References}

[1] K. I. Mohammed, M. I. Azawe, Measurement of thermal and optical properties of CR-39 solid-state nuclear detector by photothermal deflection, Nucl. Instrum. Methods Phys. Res. 308 (2013) 54-61.

[2] J. Babak, N. Atefeh, S. Nima, N. Hanieh, Surface properties of UV irradiated CR-39 polymer before and after chemical etching and registration of fingerprints on CR-39, Radiation Measurements. 101 (2017) 22-28.

[3] S. Surinder, Neerja, The effect of gamma-irradiation on the activation energy of bulk and track etching in CR-39 plastic track detector, Radiation Measurements. 42 (2007) 15071509 .

[4] Yamauchi, T. and Ichijo, H., K. Oda, Depth-dependence of the bulk etch rate of gamma-ray irradiated CR-39 track detector, Radiation Measurements. 34 (2001) 85-89.

[5] K. M. Thabayneh, M. Y. Shoeib, Studying some properties of CR-39 detector under the effect of different gamma doses, Journal of the Association of Arab Universities for Basic and Applied Sciences. 20 (2016) 55-60.
[6] A. T. Zuhair, Use of CR-39 Polymer for Radiation Dosimetry. JKAU: Eng. Sci. 22 (2011) 79-96.

[7] M. A. Malek, C. S. chang, A. Renreng, Generation of $\mathrm{CO}_{2}$ in $\gamma$ - ray irradiated CR-39 plastic, Nucl. Tracks Radiat. Meas, 35 (2002) 109-114.

[8] M. F. Zaki, Gamma-Induced Modification on Optical Band Gap of CR-39 SSNTD, Brazilian Journal of Physics. 38 (2008) 558-562.

[9] M. F. Zaki, W. A. Ghaly, H. S. El-Bahkiry, Photoluminescence, optical band gap and surface wettability of some polymeric track detectors modified by electron beam, Surf. Coat. Technol. 275 (2015) 363-368.

[10] J. Tauc, Optical Properties of Solids, Ables, A. Ed. (Amsterdam: North- Holland) (1970).

[11] A. M. Abdul-Kader, M. F. Zaki, B. A. El-Badry, Modified the optical and electrical properties of CR-39 by gamma ray irradiation, J. Radiat. Res. Appl. Sci. 7 (2014) 286-291.

[12] S. Singh, S. Prasher, The optical, chemical and spectral response of gamma-irradiated Lexan polymeric track recorder, Radiat. Meas. 40 (2005) 50-54.

[13] T. Heins, W. Enge, Oxygen effect on the etch rate in CR-39 plastic detector, Nucl. Tracks Radiat. Meas. 87 (1986) 1-6.

[14] M. F. Zaki, Y. H. Elshaer, Doaa, H. taha, Studying the Structural, Optical, Chemical and Electrochemical Etching Changes of CR-39 for Dosemetric Applications, Journal of Radiation Protection Dosimetry. 40 (2017) 1-8.

[15] T. Yamauchi, H. Nakai, Y. Somaki, K. Oda, Formation of CO2 gas and $\mathrm{OH}$ groups in CR-39 plastics due to gamma-ray and ions irradiation, Radiat. Mea. 36 (2003) 99-103. 